\title{
Prevalence of Extended Spectrum Beta- Lactamase Producers among Various Clinical Samples in a Tertiary Care Hospital: Kurnool District, India
}

\author{
Suguneswari Giddi, Sarada Dasari* and C. Suryakumari \\ Department of Microbiology, Viswabharathi Medical College, Penchikalapadu, \\ Kurnool District, A.P., India \\ *Corresponding author
}

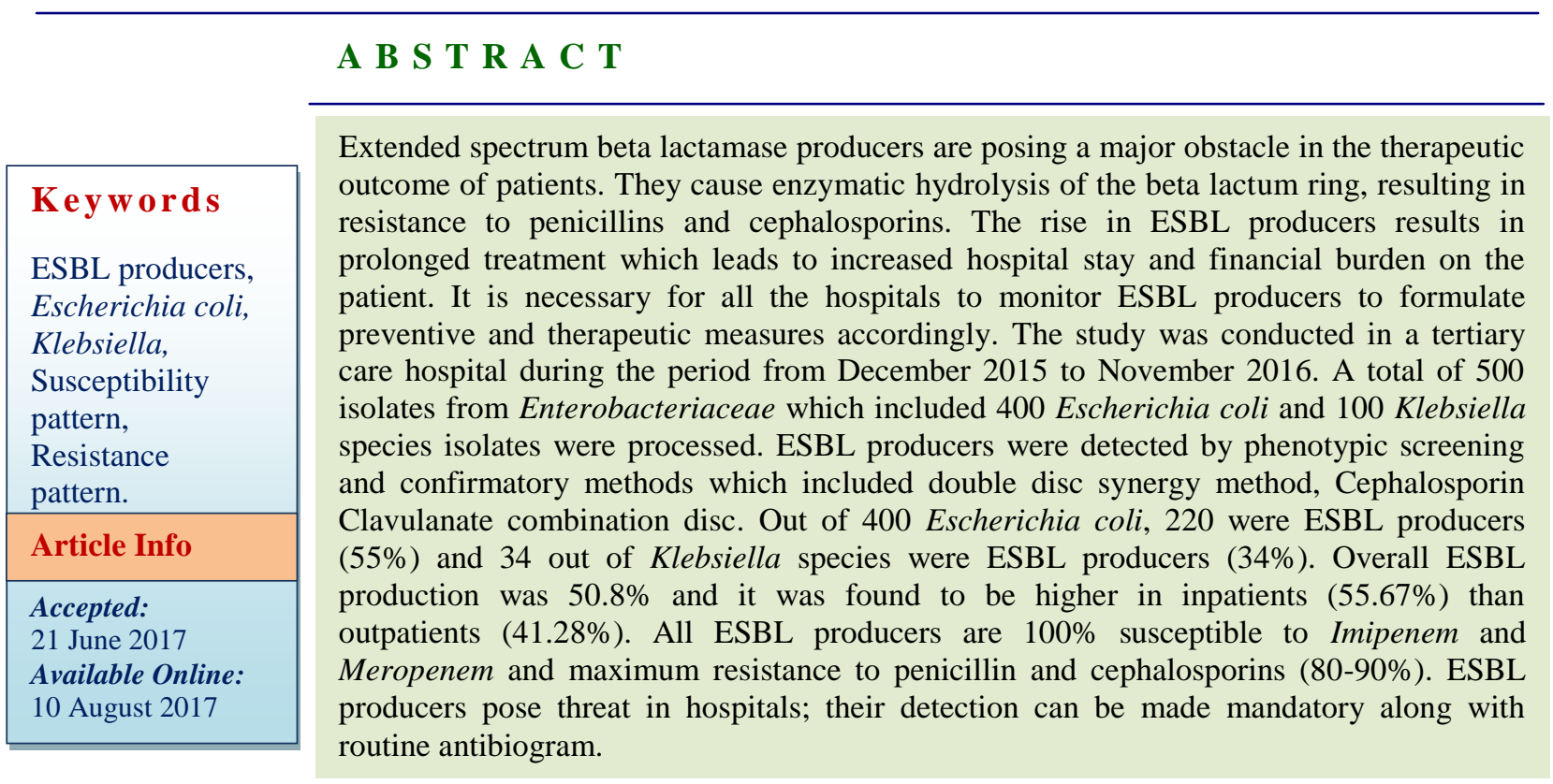

\section{Introduction}

Multidrug resistant bacteria are emerging worldwide in the hospitals and cause treatment failures especially the Enterobacteriaceae group of bacteria. Enterobacteriaceae group of organisms cause various hospital acquired infections like gastrointestinal, urinary tract and pyogenic infections. Among Enterobacteriaceae the most important member that exhibits multiple drug resistance is Escherichia coli (Dinesh Kumar et al., 2014). Enterobacteriaceae family possesses plasmid mediated $\beta$ lactamases and which is the single most important cause of resistance to penicillins and cephalosporins. This resistance is due to mutations in genes like TEM, SHV, and CTX-M. These mutations cause alteration of aminoacid configuration which results in their ability to hydrolyze various beta lactum antibiotics like penicillins, cephalosporins and monobactum (Agarwal, 2004).

Among Enterobacteriaceae the major ESBL producers are Escherichia coli and Klebsiella. ESBL producers are worldwide in distribution and its rate and methods of isolation varies in different areas. ESBL are found in clinical samples like urine, blood, sputum, swabs, 
body fluids, and catheter tips (Kenneth, 2008). Apart from resistance to broad spectrum cephalosporins and monobactums, ESBL also exhibit co-resistance to other groups of antibiotics such as quinolones, cotrimoxazole, and tobramycin, this leads to limitation of available therapeutic options. ESBL production is indicated by eight fold reduction in MIC and accentuation of zone of inhibition of third generation cephalosporins in the presence of clavulinic acid (Iraj Alipourfard, 2010).

High rate of ESBL producing organisms associated with fewer therapeutic options lead to longer hospital stay, increased cost, high rate of morbidity and mortality (Folasoge A et $a l .,, 2014)$. Therefore the present study was conducted to be able to study the prevalence, antibiotic resistance profile of ESBLs so that an effective antibiotic strategy can be planned to limit infections due to them.

\section{Materials and Methods}

The present study was conducted in Viswabharathi medical college, a tertiary care hospital in Kurnool, during the period from December 2015 to November 2016. The isolates were collected from various clinical samples which include urine, blood, sputum, swabs and body fluids. Samples from patients attending both outpatient and inpatient departments were included in the study without applying any selection criteria. Sufficient amount of various clinical samples were collected under aseptic conditions and were inoculated on to blood agar and MacConkey agar. Blood samples were collected under aseptic conditions and inoculated in brain heart infusion agar. They were subcultured on to blood agar and MacConkey agar after overnight incubation at $37^{\circ} \mathrm{C}$ for $24 \mathrm{hrs}$. All the inoculated samples were incubated at $37^{\circ} \mathrm{C}$ for $24 \mathrm{hrs}$ and were processed further for identification of the organism by studying colony morphology, motility, and gram staining and biochemical reactions.

Isolated organisms were subjected to antimicrobial susceptibility testing by KirbyBauer disc diffusion method on Mueller Hinton agar following clinical laboratory standards institute guidelines CLSI guidelines (CLSI, 2014). Commercially available antibiotic discs from Hi- media were used. The antibiotic discs used were Ampicillin $(10 \mu \mathrm{g})$, Cephalexin $(30 \mu \mathrm{g})$, Cefoxitin $(30 \mu \mathrm{g})$, Ceftazidime $(30 \mu \mathrm{g})$, Cefotaxime $(30 \mu \mathrm{g})$, Ceftriaxone $(30 \mu \mathrm{g})$, Aztreonam $(30 \mu \mathrm{g})$,

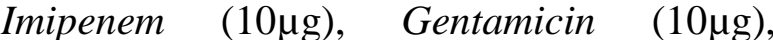

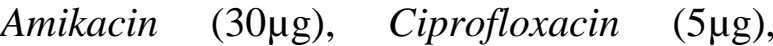

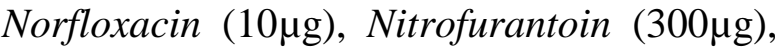

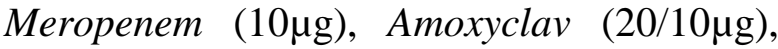
Ticarcillin-Clavulinic acid (75/10ug), Piperacillin-Tazobactum (100/10 $\mu \mathrm{g})$. ESBL detection was detected by phenotypic screening and confirmatory tests.

\section{Phenotypic screening test}

Ceftazidime, Aztreonam, Cefotaxime and Ceftriaoxone antibiotic discs were used for antibiotic sensitivity testing as a routine method. The zone of inhibition was measured and it was $\leq 22 \mathrm{~mm}$ for Ceftazidime, $\leq 27 \mathrm{~mm}$ Aztreonam, $\leq 25$ Ceftriaxone, and $\leq 27 \mathrm{~mm}$ for Cefotaxime. The above zone sizes indicate suspected ESBL producers. They were confirmed by confirmatory tests.

\section{Phenotypic confirmatory tests 2 methods}

\section{Double disk synergy test (DDS)}

It is a diffusion test in which $30 \mu \mathrm{g}$ of Ceftriaxone, Ceftazidime, Cefotaxime and Aztreonam antibiotic discs are subjected to antibiotic susceptibility testing on Mueller Hinton agar with lawn culture of the isolate about $30 \mathrm{~mm}$ from Amoxicillin Clavulanic 
acid disk. After overnight incubation extension of zone of inhibition of antibiotic disc towards Clavulanic acid containing disc indicates ESBL (Dinesh Kumar, 2014).

\section{Combination disc containing clavulanic} acid

Cefotaxime and Ceftazidime disc with and without Clavulanic acid were placed at appropriate distance on Mueller Hinton agar plate with isolate and read after overnight incubation. A difference of more than $5 \mathrm{~mm}$ of zone size between cephalosporin disc and their clavulanate containing disc indicates ESBL production (Dinesh Kumar et al., 2014).

\section{Results and Discussion}

This study was conducted in a tertiary care hospital at Kurnool district during the period December 2015 to November 2016. Samples from inpatients and outpatients attending various clinical departments are included in the study. Various clinical samples included in the study were urine, sputum, blood, pus, swabs and fluids. Maximum ESBL productions was seen in urine $(65.35 \%)$ samples followed by swabs (12.59\%), blood $(8.66 \%)$, fluid $(7.87 \%)$, sputum $(5.51 \%)$ (Table 1).

Among 254 total ESBL producers Escherichia coli was the dominant organism from urine $(96.38 \%)$, swab $(81.25 \%)$, blood $(81.81 \%)$, fluids $(70 \%)$, where as in Klebsiella maximum ESBL production was seen in sputum samples $(85.71 \%)$ (Table 2).

Out of 500 isolates, 400 isolates were Escherichia coli and 100 isolates were Klebsiella spp. Among 400 isolates of Escherichia coli 220 (55\%) were ESBL producers and $60(21.42 \%)$ were ESBL non producers. Among 100 isolates of Klebsiella species $34(34 \%)$ were ESBL producers and $36(48.57 \%)$ were non ESBL producers (Table 3).

The overall ESBL producers from total 500 samples were $50.8 \%$ and non ESBL producers were $19.2 \%$ (Table 4 ).

All the isolates exhibited $100 \%$ sensitivity to Imipenem and Meropenem. Among all the isolates $80.31 \%$ showed sensitivity to Pipercillin-Tazobactum, $77.16 \%$ to Amikacin and Amoxyclav, followed by $76.37 \%$ to Ticarcillin-Clavulanic acid, $58.26 \%$ to Gentamicin and $50.39 \%$ isolates were sensitive to Nitrofurantoin. All the isolates showed maximum resistance $(80 \%$ to $90 \%)$ to all 4- generation Cephalosporins.

The resistance pattern for other drugs were 93.7\% to Ampicillin, $90.55 \%$ to Aztreonam, $72.64 \%$ to Norfloxacin, and $70.07 \%$ isolates were resistant to Ciprofloxacin (Table 6).

Table.1 Frequency of samples yielding ESBL producers

\begin{tabular}{|l|l|l|}
\hline Source & Frequency & Percentage \\
\hline Urine & 166 & $65.35 \%$ \\
Swabs & 32 & $12.59 \%$ \\
Blood & 22 & $8.66 \%$ \\
Fluids & 20 & $7.87 \%$ \\
Sputum & 14 & $5.51 \%$ \\
\hline Total & 254 & \\
\hline
\end{tabular}

ESBL- Extended spectrum betalactamase 
Table.2 ESBL prevalence among various clinical samples

\begin{tabular}{|l|l|l|l|l|l|}
\hline Sample & No of isolates & \multicolumn{3}{|l|}{ ESBL Positive } & \multicolumn{2}{l|}{ Klebsiella } \\
\cline { 3 - 6 } & & \multicolumn{2}{|l|}{ Escherichia coli } & No & Percentage \\
\hline & & No & Percentage & No & $3.61 \%$ \\
\hline Urine & 166 & 26 & $96.38 \%$ & 6 & $18.75 \%$ \\
Swabs & 32 & 18 & $81.25 \%$ & 6 & $18.18 \%$ \\
Blood & 22 & 14 & $81.81 \%$ & 4 & $30 \%$ \\
Fluids & 20 & 2 & $70 \%$ & 6 & $85.71 \%$ \\
Sputum & 14 & 220 & $14.28 \%$ & 12 & \\
\hline Total & 254 & & 34 & \\
\hline
\end{tabular}

ESBL- Extended spectrum betalactamase

Table.3 ESBL production in Escherichia coli and Klebsiella

\begin{tabular}{|l|l|l|l|l|l|l|l|l|}
\hline & \multicolumn{2}{|l|}{ Escherichia coli } & \multicolumn{2}{l|}{ Klebsiella } \\
& $\begin{array}{l}\text { E.coli } \\
\text { isolates }\end{array}$ & $\begin{array}{l}\text { Screening } \\
\text { positives }\end{array}$ & $\begin{array}{l}\text { ESBL } \\
\text { producers }\end{array}$ & $\begin{array}{l}\text { Non- } \\
\text { ESBL } \\
\text { producers }\end{array}$ & $\begin{array}{l}\text { Klebsiella } \\
\text { isolates }\end{array}$ & $\begin{array}{l}\text { Screening } \\
\text { positives }\end{array}$ & $\begin{array}{l}\text { ESBL } \\
\text { producers }\end{array}$ & $\begin{array}{l}\text { Non- } \\
\text { ESBL } \\
\text { producers }\end{array}$ \\
\hline Urine & 250 & 193 & 160 & 33 & 17 & 14 & 6 & 8 \\
Swabs & 85 & 37 & 26 & 11 & 13 & 9 & 6 & 3 \\
Blood & 30 & 27 & 18 & 9 & 10 & 8 & 4 & 4 \\
Fluids & 25 & 19 & 14 & 5 & 16 & 11 & 6 & 5 \\
Sputum & 10 & 4 & 2 & 2 & 44 & 28 & 12 & 16 \\
\hline & 400 & 280 & $220(55 \%)$ & 60 & 100 & 70 & $34(34 \%)$ & 36 \\
& & & & $(21.42 \%)$ & & & & \\
\end{tabular}

E.coli- Escherichia coli, ESBL-Extended spectrum betalactamase, Non-ESBL- Non Extended spectrum betalactamase

Table.4 ESBL and Non ESBL producers in Escherichia coli and Klebsiella

\begin{tabular}{|l|l|l|l|}
\hline Organisms & Total isolates & ESBL producers \& \% & ESBL non-producers \& \% \\
\hline Escherichia coli & 400 & $220(55 \%)$ & $60(21.42 \%)$ \\
\hline Klebsiella & 100 & $34(34 \%)$ & $36(48.57 \%)$ \\
\hline Total & 500 & $254(50.8 \%)$ & $96(19.2 \%)$ \\
\hline \multicolumn{2}{|l|}{} & P- Value $<0.01$ \\
\hline
\end{tabular}

ESBL- Extended spectrum betalactamase, Non-ESBL- Non Extended spectrum betalactamase

Table.5 Number of Enterobacteriaceae isolates in in-patients and out-patients

\begin{tabular}{|l|l|l|l|l|l|}
\hline \multirow{2}{*}{ Specimen } & \multicolumn{2}{|l|}{ Escherichia coli } & \multirow{2}{*}{ Klebsiella } \\
\cline { 2 - 3 } \cline { 5 - 6 } & IP & OP & Specimen & IP & OP \\
\hline Urine $(\mathrm{n}=250)$ & 147 & 103 & Urine $(\mathrm{n}=17)$ & 10 & 7 \\
Swabs $(\mathrm{n}=85)$ & 44 & 57 & Swabs $(\mathrm{n}=13)$ & 7 & 6 \\
Sputum $(\mathrm{n}=30)$ & 2 & 8 & Sputum $(\mathrm{n}=44)$ & 28 & 16 \\
Blood $(\mathrm{n}=30)$ & 20 & 10 & Blood $(\mathrm{n}=10)$ & 8 & 2 \\
Fluids $(\mathrm{n}=25)$ & 4 & 11 & Fluids $(\mathrm{n}=16)$ & 12 & 4 \\
\hline 400 & 217 & 183 & 100 & 65 & 35 \\
\hline \multicolumn{7}{|l|}{} \\
\hline
\end{tabular}

IP- In patient, OP- Outpatient 
Table.6 Susceptibility and resistance pattern of ESBL producers

\begin{tabular}{|l|l|l|l|l|}
\hline Antibiotic & Sensitivity & Pescentage & Number & Percentage \\
\hline & Number & $6.24 \%$ & 238 & $93.7 \%$ \\
\hline Ampicillin & 16 & $16.53 \%$ & 212 & $83.46 \%$ \\
Cephalexin & 42 & $19.68 \%$ & 204 & $80.3 \%$ \\
Cefoxitin & 50 & $14.56 \%$ & 217 & $85.43 \%$ \\
Ceftazidime & 37 & $9.44 \%$ & 230 & $90.55 \%$ \\
Cefotoxime & 24 & $11.81 \%$ & 224 & $88.18 \%$ \\
Ceftriaxone & 30 & $12.59 \%$ & 222 & $87.40 \%$ \\
Cefaperazone & 32 & $9.44 \%$ & 230 & $90.55 \%$ \\
Aztreonam & 24 & $100 \%$ & 0 & 0 \\
Imipenem & 254 & $58.26 \%$ & 106 & $41.73 \%$ \\
Gentamicin & 148 & $77.16 \%$ & 58 & $22.83 \%$ \\
Amikacin & 196 & $29.92 \%$ & 178 & $70.07 \%$ \\
Ciprofloxacin & 76 & $33.07 \%$ & 170 & $72.64 \%$ \\
Norfloxacin & 84 & $50-39 \%$ & 126 & $49.60 \%$ \\
Nitrofurantoin & 128 & $100 \%$ & 0 & 0 \\
Meropenem & 254 & $77.16 \%$ & 58 & $22.83 \%$ \\
Amoxyclav & 196 & $76.37 \%$ & 60 & $23.62 \%$ \\
Ticarcillin- & 194 & & & \\
Clavulinic acid & & $80.31 \%$ & 50 & $19.16 \%$ \\
Pipercillin- & 204 & & & \\
Tazobactum & & & & \\
\hline
\end{tabular}

ESBL- Extended spectrum betalactamase

A total of 500 isolates from Enterobacteriaceae were processed for ESBL production in our one year study period. Although other members of enterobacteriacae also produce ESBL, Escherichia coli and Klebsiella species were taken into consideration.

Out of the 400 Escherichia coli isolates 220 $(55 \%)$ were ESBL producers, 34 (34\%) among 100 Klebsiella species were ESBL producers. Overall ESBL production was seen in 254 isolates $(50.84 \%)$ which matches with the study by Hima bindu (2015) (58.8\%). ESBL production in Escherichia coli and Klebsiella was 55\% and 34\% respectively in our study which is consistent with studies by Hima bindu (2015) $(56.4 \%, 62.3 \%)$ and Sasirekha $(52.8 \%, 45.1 \%)$. Study by Dinesh Kumar et al., (2014) showed $55.55 \%$ of ESBL production in Escherichia coli which is highly similar with our study (55\%). ESBL production in Klebsiella species in our study was $34 \%$ which is similar to the study by Shukla et al., (30.18\%) and slightly less than the study done by Baby Padmini et al., (2004) $(40 \%)$.

Overall percentage of ESBL producers in India varies between 22-75\% (Aruna et al., 2012). In our study ESBL production was found to be high among inpatients (55.67\%) than out patients(48.28\%) this can be compared to the study by Dinesh Kumar et al., (2014) (IP-60.95\%, OP-48\%) and Rinki et al., (2016) (IP-71.3\%, OP-50.5\%). The present study showed highest number of ESBL producers from urine and Escherichia coli was found to be the dominant organism, this observation is in agreement with the study by Folasoge.A.et al.,, In our study highest susceptibility was found for Imipenem and Meropenem (100\%) followed by Piperacillin-Tazobactum (80\%), Amikacin, 
Amoxyclav (77.16\%) and TicarcillinClavulanate $(76 \%)$. Maximum resistance was seen for Ampicillin, all 4-generations of Cephalosporins and Aztreonam (80-90\%). This susceptibility and resistance pattern is in accordance with other studies (Pooja Shakya et al., 2017; Akila et al., 2016 and Dinesh Kumar, 2014).

The present study was done in a tertiary care hospital at Kurnool district on ESBL production among Enterobacteriaceae group of organisms. Escherichia coli emerged as a dominant ESBL producer when compared to Klebsiella. All 4 generations of Cephalosporins, and other drugs like Ampicillin, Aztreonam and Ciprofloxacin showed maximum resistance; only Imipenem and Meropenem exhibited 100\% susceptibility to ESBL producers. Indiscriminate usage of board spectrum antibiotics as an option of empirical choice of treatment leads to rise of ESBL producers complicating treatment options. Strict implementation of hospital infection control measures along with restricting inadvertent use of cephalosporins may contribute in preventing ESBL producers.

\section{References}

Akila. K., et al., 2016. Prevalence of ESBL producing Klebsiella species and their in-vitro antimicrobial susceptibility pattern in a tertiary care hospital. IOSR Journal of Dental and Medical Sciences, 5-10.

Aruna. K., et al., 2012. Prevalence of ESBL production among uropathogens in South Mumbai and its Antibiogram pattern. EXCLIJ. 11:363-372.

Baby Padmini, et al., 2004. Extended spectrum beta-lactamases in urinary isolates of Escherichia coli and Klebsiella pneumoniae - prevalence and susceptibility pattern in a tertiary care hospital. Indian J Med Microbiol, 22, 172-174

Chowdary U., et al., 2004. Extended spectrum $\beta$-alactamases (ESBL);An Emerging threat to clinical therapeutics. Indian journal of medical microbiology, 22(2): $75-80$.

CLSI, 2014. Performance Standards for Antimicrobial Susceptibility Testing; Twenty-Fourth Informational Supplement. CLSI document M100S24. Wayne, PA: Clinical and Laboratory Standards Institute.

Dalela. G., 2012. Prevalence of ESBL produces among Gram negative bacilli from various clinical isolates in a tertiary care hospital at Jhalawar, Rajasthan, India. Journal of Clinical Diag.Res. 6(2):182-187.

Dinesh Kumar. A., et al., 2014. Antimicrobial susceptibility profile of extended spectrum betalactamse producing Escherichia coli from various clinical samples. Infectious diseases reserch and treatment.

Folasoge, A., et al., 2014. A multicenter study of Beta Lactamase resistant Escherichia coli and Klebsiella pneumoniae reveals high level chromosome mediated extended spectrum $\beta$ Lactamase resistance in Ogun State, Nigeria. Interdisciplinary perspectives on infectious diseases.

Hima Bindu. M., et al., 2015. Prevalence of ESBL production in Escherichia coli and Klebsiella Spp from different clinical samples-A study in a teaching hospital in Telangana, India. International Journal of Current Microbiology and Applied Sciences, 4(3):236-243.

Iraj Alipourfard, et al., 2010. Antibiogram of extended spectrum Beta-lactamase producing Escherichia coli and Klebsiella pneumoniae isolated from hospital samples. Bangladesh journal of 
medical mecrobiology, vol 4. (01):3236.

Kenneth. T., 2008. Bacterial Resistance to Antibiotics. Todars Online Textbook of Bacteriology.

Pooja Shakya, et al., 2017. ESBL production among E.coli and Klebsiella Spp causing urinary tract infections: A hospital based study. The Open Microbiology Journal, 11, 23-30.

Rinki R., et al., 2016. The detection of extended spectrum Beta-Lactamases (ESBLs) producing Escherichia coli isolated from clinical samples. International journal of advance research in biological sciences, 3(5):915.

Sasirekha. B., 2013. Prevalence of ESBL, Amp C $\beta$-Lactamases and MRSA among uropathogens and its antibiogram. EXCLIJ, 12:81-88.

Shukla. R., 2004. Prevalence of extended spectrum beta-lactamase producing Klebsiella pneumoniae in a tertiary care hospital. Indian J Med Microbiol, 22, 87-91.

\section{How to cite this article:}

Suguneswari Giddi, Sarada Dasari and Suryakumari, C. 2017. Prevalence of Extended Spectrum Beta- Lactamase Producers among Various Clinical Samples in a Tertiary Care Hospital: Kurnool District, India. Int.J.Curr.Microbiol.App.Sci. 6(8): 2857-2863. doi: https://doi.org/10.20546/ijcmas.2017.608.341 